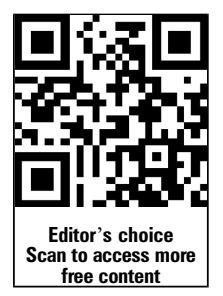

\title{
Injury and illness definitions and data collection procedures for use in epidemiological studies in Athletics (track and field): Consensus statement
}

\author{
Toomas Timpka, ${ }^{1}$ Juan-Manuel Alonso, ${ }^{2,3}$ Jenny Jacobsson, ${ }^{1,4}$ Astrid Junge ${ }^{5,6,7}$ \\ Pedro Branco, ${ }^{2,8}$ Ben Clarsen, 9, 10 Jan Kowalski, 1,4,11,12 Margo Mountjoy, ${ }^{13,14,15}$ \\ Sverker Nilsson, ${ }^{1,4}$ Babette Pluim, ${ }^{16}$ Per Renström, ${ }^{12,13}$ Ola Rønsen, ${ }^{2,10}$ \\ Kathrin Steffen, ${ }^{9}$ Pascal Edouard ${ }^{17,18,19}$
}

- Additional material is published online only. To view please visit the journal online (http://dx.doi.org/10.1136/ bjsports-2013-093241)

For numbered affiliations see end of article.

\section{Correspondence to}

Dr Juan-Manuel Alonso, Sports Medicine Department, Aspetar Qatar Orthopedics and Sports Medicine Hospital, PO Box 29222, Doha, Qatar; juan-manuel.alonso@aspetar. com

Accepted 31 January 2014

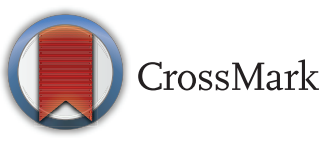

To cite: Timpka T, Alonso J-M, Jacobsson J, et al. Br J Sports Med 2014;48:483-490.

\section{ABSTRACT \\ Background Movement towards sport safety in} Athletics through the introduction of preventive strategies requires consensus on definitions and methods for reporting epidemiological data in the various populations of athletes.

Objective To define health-related incidents (injuries and illnesses) that should be recorded in epidemiological studies in Athletics, and the criteria for recording their nature, cause and severity, as well as standards for data collection and analysis procedures.

Methods A 1-day meeting of 14 experts from eight countries representing a range of Athletics stakeholders and sport science researchers was facilitated. Definitions of injuries and illnesses, study design and data collection for epidemiological studies in Athletics were discussed during the meeting. Two members of the group produced a draft statement after this meeting, and distributed to the group members for their input. A revision was prepared, and the procedure was repeated to finalise the consensus statement.

Results Definitions of injuries and illnesses and categories for recording of their nature, cause and severity were provided. Essential baseline information was listed. Guidelines on the recording of exposure data during competition and training and the calculation of prevalence and incidences were given. Finally, methodological guidance for consistent recording and reporting on injury and illness in athletics was described. Conclusions This consensus statement provides definitions and methodological guidance for epidemiological studies in Athletics. Consistent use of the definitions and methodological guidance would lead to more reliable and comparable evidence.

\section{INTRODUCTION}

Athletics (track and field) is a global sport and the largest at the Olympics Games, contributing about $20 \%$ of all participants (http://www.olympic.org). ${ }^{1-5}$ Athletics includes the track and field disciplines of track running, jumping and throwing, race walking and cross-country and road running. The sport is governed by the International Association of Athletics Federation (IAAF; http://www.iaaf.org). World Outdoor Championships are held every second year at which almost 2000 athletes representing all continents compete. ${ }^{1-3}$ From clinical experience, we know that competition and training in Athletics poses considerable risks of injuries and illnesses. Despite this, few studies have prospectively reported injury and illness incidences and patterns in populations of athletes representing all groups of events over the course of an entire season or longer. $^{6-8}$

In 2007 , the IAAF introduced routine data collection on injury incidence and characteristics during the World Championships. ${ }^{1}$ Since 2008 , injury surveillance has also included the corresponding competitions during the Olympic Games. ${ }^{4} 5$ Collection of data on illness was added from 2009. ${ }^{2}$ Recently, health surveillance has also been introduced at competitions organised by European Athletics (EAA). ${ }^{9} 10$ Data from these championship settings showed that approximately $10 \%$ of athletes sustained an injury and $7 \%$ suffered an illness during the relative short period of competition. 23910 While studies performed at high-level competition provide essential information, they are not sufficient for understanding the general injury and illness risks during a longer time period and across populations of athletes. The exposure to injury and illness risks during a championship is not representative for the entire season, as the training performed during the time period leading up to major competitions is not comparable with the training during the rest of the year. Older studies of club and elite athletic cohorts have reported that Athletics injuries were mostly observed during training with annual cumulative injury incidence ranging between $61 \%$ and $75 \% .^{7}{ }^{11}$ Recent studies from Swedish Athletics found that almost every second adult athlete experiences a performance-limiting musculoskeletal injury, primarily of the overuse type, during 1 year. ${ }^{12}$ Furthermore, the Swedish data also showed that a training load index combining increased training hours and intensity with a history of severe injury the previous year was a positive predictor for the risk of injury. ${ }^{6}$ The scientific literature published on Athletics injury and illness epidemiology in the past 10 years thus provides more information from short-term surveillance during competitions ${ }^{1-3} 9{ }^{10}$ than prospective studies covering complete seasons, ${ }^{6}{ }^{13}$ and none of the long-term studies have reported illness data. Hence, there is a lack of information on injury and illness prevalence and risks in athletes during the entire season. This mismatch could be, in part, due to the difficulty in conducting injury and illness surveillance in individual sports especially over an entire athletic season. 
Consensus statements on sports injury definitions and data collection procedures have been published for several team sports (eg, football ${ }^{14}$ and rugby ${ }^{15}$ ), and recently for individual sports, (eg, tennis ${ }^{16}$ and thoroughbred horse racing ${ }^{17}$ ). To ensure the development of a safe sporting environment in Athletics, it is essential to reach a similar agreement on injury and illness definitions and to use a standardised methodological approach to data collection and analysis so that results can be compared or combined. This requires establishment of consensus definitions within Athletics and extending the consensus procedure to involve representatives from team sports and other individual sports to allow comparisons. The aim of this consensus statement was therefore twofold: (1) to define health-related incidents (injuries and illnesses) that should be recorded in epidemiological studies in Athletics, as well as their nature, causes and severity and (2) to provide guidance on important methodological aspects with respect to the epidemiological studies in Athletics also taking into consideration procedures used in other sports and relevant international standards.

\section{CONSENSUS PROCEDURE}

TT, JJ, PB and J-MA initiated the Athletics Consensus Conference on the occasion of the European Indoors Championships in Athletics. Several national and international Athletics governing bodies as well as renowned researchers in the field were assembled into an Athletics Consensus Group with the mission to develop a consensus statement for future studies of Athletics injuries and illnesses. Previous consensus methodology was reviewed ${ }^{14-18}$ and problems with analysing and reporting epidemiological data in Athletics were identified. Based on the identified problems and needs for change, basic requirements for reporting of epidemiological data in Athletics were delineated. The consensus procedure involved the following steps:

- Key people were identified and invited to represent following groups: (1) clinical professionals with experience from Athletics or other sports as team physicians/physiotherapists; (2) the IAAF, other international sports federations and the Medical Commission of the International Olympic Committee (IOC); (3) researchers in the field of sports injuries epidemiology, ideally with previous involvement in consensus statements in other sports and (4) editorial boards of major scientific journals in sports medicine. The number and selection of invitations was also restricted by limited meeting funding, practical group process considerations and geographical vicinity to the meeting venue. Invitations were sent to 16 experts; 2 rejected the invitation due to other commitments.

- A person with social science training was appointed to moderate the consensus meeting.

- An agenda and a resume of needs and requirements on epidemiological reporting in Athletics (box 1) were circulated to all members of the group to provide the basis for discussions, and participants were invited to identify any additional topics to be added to the agenda.

- During the consensus meeting, each topic was debated and discussed openly.

- The moderator ensured that all delegates were provided the opportunity to present their opinions on each topic, and the deliberations were limited to the agreed timetable of the agenda. If needed, on conclusion of the discussion on any topic, the moderator called either for a consensus of all participants or for a vote on proposals.

The consensus meeting with 14 participating experts from eight countries took place on 4 March 2013 in Gothenburg, Sweden. Twelve members of the Athletics Consensus Group were present at the meeting in person and 2 members took part via a video conference (J-MA and $\mathrm{MM}$ ). The meeting was videotaped for documentation purposes. Following the consensus meeting, a draft manuscript was prepared by the two leading authors (TT and J-MA) based on the review of previous consensus statements ${ }^{14-18}$ and the agreements were recorded during the meeting. This document was distributed three times to two coauthors (JJ and PE). Then, an updated version of the manuscript was circulated to all group members with an appeal for comments to be returned within a dateline. All remarks received were reviewed by the two lead authors and, where applicable, incorporated into revisions of the manuscript. Where a suggested change was not incorporated, a written explanation was prepared and distributed to group members. This method was repeated two times. After the final iteration, all members of the group were asked to confirm their agreement with this final statement version. Disagreements with the consensus were recorded and documented (see online text supplement 1).

\section{THE CONSENSUS STATEMENT}

The definitions and guidelines presented in this report are based on previous consensus statements on epidemiological reporting of injury and illness in sports. ${ }^{14-16} 18$ Our recommendations focus on and reflect the specific issues facing Athletics (box 1) at international and domestic levels.

\section{Definitions}

Recordable health-related incidents (injury/illness)

A recordable health-related incident in Athletics is defined as

Any physical or psychological complaint or manifestation experienced by an athlete, irrespective of the need for medical attention or time loss from Athletics activities.

Recordable incidents resulting from participation in Athletics competition and training should be included in surveillance studies, and incidents that are probably not directly related to Athletics may also be recorded, but these should be analysed separately. The term 'incident' was adopted in this statement, rather than 'injury' or 'medical condition', ${ }^{14-16} 18$ in order to highlight the desire to also collect syndromic (prediagnostic) data and overuse injuries. As in the Tennis consensus statement, ${ }^{16}$ the term 'manifestation' was added to the term 'complaint', due to the fact that athletes do not always feel or experience a medically recognised condition. However, an athlete may have external signs that would alert a health professional to a condition, such as a skin lesion or osteophyte. The term 'psychological' was transferred from the Tennis consensus report, ${ }^{16}$ as overtraining and burnout may occur also in Athletics. Also following Tennis Consensus examples, ${ }^{16}$ sunrelated skin conditions and exercise-induced asthma could be recorded as resulting from participation in Athletics. However, road traffic incidents and domestic accidents should not be taken into account.

\section{Injury}

An Athletics injury is defined as: 'A physical complaint or observable damage to body tissue produced by the transfer of energy experienced or sustained by an athlete during participation in Athletics training or competition, regardless of whether it received medical attention or its consequences with respect to impairments in connection with competition or training. ,2 519 
End-user needs associated with collection of epidemiological data in Athletics:

Need 1: Overuse injuries-Overuse injuries are the main cause of impaired performance and time loss injuries during training and competition in Athletics. To prevent overuse injuries, data must be collected prospectively and during a complete season, also in young athletes.

Need 2: Classifications-Current classifications in sports epidemiology need to be discussed and suitably adapted for application in Athletics, for example, clarification of 'overuse with sudden onset' vs 'non-contact trauma'; 'new injuries' vs 'acute injuries'.

Need 3: Recognition of Athletics as an individual sport. There are inherent features associated with the nature of Athletics as a sport that need to be addressed in data collection: athletic (track and field) clubs are not professional and they rarely have a single medical team and/or medical team employed by the club. There are also significant differences between countries. Athletes located far from the club facilities (various geographic locations; for professional, training or familial reasons). Training can be either individual or in groups, and may have their own medical support structure, which could lead to difficulties coordinating medical services and injury and illness surveillance.

Need 4: Illness codes-The current International Olympic Committee (IOC) illness codes need to be adjusted to be more systematically and consistently with international illness coding in order to facilitate better knowledge of illnesses in Athletics.

Need 5: Multidisciplinary perspectives-Clinicians, Athletics associations, coaches and athletes may have different understandings on what is recordable injury or illness in Athletics, all of them equally legitimate to take into account for epidemiological reporting and for generating relevant intervention planning information. All fitting definitions need to be formulated to reduce bias and errors.

Need 6: Compatibility—Epidemiological coding and reporting in Athletics need preferably to be compatible with systems and procedures in other areas of epidemiological research, and not restricted to sports only.

\section{Requirements on data collection in Athletics}

Requirement 1: Focus on overuse injuries-Injurious events caused by isolated exposure to physical energy ('injuries') and repeated bouts of load or prolonged bouts of exposure to causal mechanism ('overuse injuries and diseases') differ in aetiology and require different clinical and preventive interventions in Athletics. These categories of events must be classified and analysed separately.

Requirement 2: Multiple parallel perspectives-Health service, sports body, coach and athlete views on injuries and illness constitute separate and valid perspectives on reporting epidemiological data in Athletics. All these perspectives must be taken into account while building a system for epidemiological reporting.

Requirement 3: Feasibility in longitudinal studies-Reporting of epidemiological data in Athletics should be feasible at championships and in longitudinal prospective studies.

Requirement 4: Applicability to young age and gender-Reporting of epidemiological data in Athletics must apply to adult and youth athletes (including procedures for parental consent) as well as to both genders.

Requirement 5: Adaptation to individual sports-Reporting of epidemiological data in Athletics must take into account that Athletics is an individual sport and that physicians and other medical staff may not always be available to record the initial incidents of injury or illness.

Requirement 6: Compatibility with electronic patient records systems-Collection of epidemiological data from athletes preferably.

\section{Illness}

An Athletics illness is defined as: "A physical or psychological complaint or manifestation by an athlete not related to injury, regardless of whether it received medical attention or its consequences with respect to impairments in connection with competition or training." 519

\section{Medical attention}

Medical attention refers to an assessment of an athlete's medical condition by a qualified medical/healthcare practitioner, such as a physician, physiotherapist, nurse, osteopath, chiropractor or qualified athletic trainer (in the USA). A field request by an athlete for medical attention, for example, for adjustment of a knee taping, should not be recorded as an 'incident' unless it is identified as a treatable condition with evidence of aggravation of the athlete's existing knee condition. A medical-attention definition has most often been employed in studies of injuries and illnesses during major international competitions such as the Olympic Games and Athletics championships where all athletes have similar access to medical care. ${ }^{1-5} 1920$ However, it should be recognised that in studies where athletes' medical coverage is non-uniform, the use of a medical attention definition may lead to systematic bias in surveillance data. ${ }^{21}$

\section{Time loss}

"A time loss injury or illness is one that leads to the athlete being unable to take full part in athletics training and/or competition the day after the incident occurred." 141518 It should be noted that studies applying a time loss definition can miss a large share of overuse conditions. ${ }^{22-24}$

\section{Severity of incidents}

In prospective studies of injury incidence, the severity of a recordable incident is defined as:

The number of days that have elapsed from the day after the onset of the incident to the day of the athlete's return to full participation in Athletics training and become fully available for competition. ${ }^{14}$

The severity is thus determined by the number of days of absence from full Athletics participation. The day on which an incident first occurs does not count when determining injury 
severity; the assessment of severity (days lost) starts on the following day if the athlete is unable to take part in full and/or normal training and/or competition. Severity should be reported as minor (1-7 days) which could be subdivided into slight ( 1 day), minimal ( $2-3$ days), mild ( $4-7$ days), moderately serious (8-28 days), serious ( $>28$ days -6 months) or long-term (>6 months). ${ }^{1425}$

Some athletes can continue sports without using the injured body part or by adjusting the exposure to the injured body part, although participation is not full.

When studies aim to monitor the prevalence of injury and illness problems and their consequences, the use of alternative methods of measuring the severity of incidents is relevant, such as the score proposed by Clarsen et al. ${ }^{23}{ }^{24}$ In particular, this approach can be used to monitor the progress of overuse injuries over time.

\section{Full recovery}

In many Athletics events, competing is seldom possible without full function, and a period of unrestricted training is usually required beforehand. However, many sudden and gradual-onset incidents develop into chronic conditions, with athletes returning to training while still experiencing adverse effects. Thus, a common notion of full recovery is important to enable the calculation of severity and the classification of recurrent incidents. $^{25}$ Ideally, recovery should be determined by expert medical opinion. However, the lack of uniform medical coverage may be a factor limiting the feasibility of this approach in athletics studies. Therefore, 'return-to-play' guidelines should be used, whereby full recovery is defined as 'the return to full athletics training and (availability for) competitions'. Consequently, the ability to train or compete ('return-to-play') in most events of Athletics assumes full recovery and normal function.

The normal function and participation in Athletics may be evaluated using performance during competitions as a reference, as well as the type/mode, volume and intensity of training that can be completed without symptoms.

\section{Recurrent conditions}

In congruence with previous consensus statements in different sports, ${ }^{14-16} 20$ a recurrent condition qualifies as a recordable incident if defined as

An incident of the same type and at the same site linked to an index incident and which occurs after an athlete's return to full function and participation ("full recovery") from the index recordable incident.

Following Fuller et al's ${ }^{26}$ framework for recording recurrences, reinjures and exacerbations in injury surveillance, an index incident is defined as the first recorded incident in a series of incidents constituting a recurrent condition. Recurrent conditions should be subcategorised into reinjuries and exacerbations. A reinjury is a repeat episode of a fully recovered index injury. An exacerbation is worsening in the state of a non-recovered index injury or illness. ${ }^{26}$

Overuse conditions involving continual or sporadic experiences of pain or other physical discomfort should be analysed separately. ${ }^{27}$

For example, an athlete may rapidly develop pain, and miss 2 weeks of normal training before returning to normal (competition-level) training while still experiencing pain; some 6 weeks later the pain level increases to such an extent that the athlete misses a further 4 weeks of normal training. The first episode should be recorded as a sudden onset incident with a severity of 14 days, while the second episode should be recorded as an exacerbation with a severity of 28 days. ${ }^{26}$

\section{Subsequent injuries}

Recordable incidents in Athletics are often succeeding or recurrent. In particular, injuries (of either the same or a different type) can be strongly influenced by a previous injury. Therefore, correct categorising of subsequent injuries (multiple, recurrent, exacerbation or new) before the analysis is essential (eg, using the SIC model). ${ }^{27}$ The categorisation of subsequent injuries needs to take into account the different modes of onset of injuries and the dependency structures between injury types. Also, it is important to declare the analytical assumptions regarding the repeated injuries, whether or not they are in a distinct order and are competing events. ${ }^{27}$

\section{Details of recordable incidents}

Injuries should be described according to the affected body part (table 1), side, type (box 2), mode of onset and cause (table 2), date of injury incurrence and of full return to Athletics practice, and the designation of the injury as an index incident or a recurrence. In addition, the activity circumstances of the injury should be recorded; for example, in-competition (heat, round, final) or training. Any incident experienced during the warm-up period in association with competition should be recorded as a competition incident. If the aim of a study was to analyse the incidence of injury associated with specific conditions (eg, training activities and capabilities), then these specific conditions have to be recorded for all injuries.

Illnesses should be recorded using prediagnostic categories for affected system (box 3), main symptoms (box 4) and mode of onset and causes (table 3).

Health professional should use sport-specific codes, such as the Orchard Sports Injury Classification System and the Sports

Table 1 Injury body part (main groupings and subcategories for location of incidents modified from references 1, 2 and 18)

\begin{tabular}{lllll}
\hline Head and trunk & \multicolumn{2}{l}{ Upper extremity } & Lower extremity \\
\hline 1 Face (including eye, ear, nose) & 11 & Shoulder/clavicle & 21 & Hip \\
2 Head & $12 \mathrm{a} / \mathrm{p}$ & Upper arm (anterior/posterior) & 22 & Groin \\
3 Neck/cervical spine & $13 \mathrm{a} / \mathrm{p}$ & Elbow (anterior/posterior) $13 \mathrm{~m} / \mathrm{l}$ Elbow (medial/lateral) & $23 \mathrm{a} / \mathrm{p}$ & Thigh (anterior/posterior) 24a/p Knee (anterior/posterior) \\
4 Thoracic spine/upper back & $14 \mathrm{a} / \mathrm{p}$ & Forearm (anterior/posterior) & $24 \mathrm{~m} / \mathrm{l}$ & Knee (medial/lateral) \\
5 Sternum/ribs & $15 \mathrm{a} / \mathrm{p}$ & Wrist (anterior/posterior) & $25 \mathrm{a} / \mathrm{p}$ & Lower leg (anterior/posterior) \\
6 Lumbar spine/lower back & $16 \mathrm{a} / \mathrm{p}$ & Hand (anterior/posterior) & $26 \quad$ Achilles tendon \\
7 Abdomen & $17 \mathrm{a} / \mathrm{p}$ & Finger (anterior/posterior) & $27 \mathrm{~m} / \mathrm{l}$ & Ankle (medial/lateral) \\
8 Pelvis/sacrum/buttock & $18 \mathrm{a} / \mathrm{p}$ & Thumb (anterior/posterior) & $28 \mathrm{a} / \mathrm{p}$ & Foot/toe (anterior/posterior) \\
\hline
\end{tabular}




\section{Box 2 Type of injury (modified from references 1, 2 and 18)}

1. Concussion (regardless of loss of consciousness)

2. Fracture (traumatic)

3. Stress fracture (overuse)

4. Other bone injuries

5. Dislocation, subluxation

6. Tendon rupture

7. Ligamentous rupture

8. Sprain (injury of joint and/or ligaments)

9. Lesion of meniscus or cartilage

10. Strain/muscle rupture/tear

11. Contusion/haematoma/bruise

12. Tendinosis/tendinopathy

13. Arthritis/synovitis/bursitis

14. Fasciitis/aponeurosis injury

15. Impingement

16. Laceration/abrasion/skin lesion

17. Dental injury/broken tooth

18. Nerve injury/spinal cord injury

19. Muscle cramps or spasm

20. Growth plate disturbance/avulsion

21. Other

Medicine Diagnostic Coding systems for diagnostic reports. ${ }^{28} 29$ The International Classification of Diseases and Injuries (ICD or ICPC) may be considered, in particular for studies performed in community settings. ${ }^{30}$ The International Classification of Functioning (ICF) can be considered for studies using the time loss definition of recordable incidents. ${ }^{31}$ If health-related data are recorded by a coach or an athlete, the recordings should be reviewed by a health professional.

\section{Mode of onset}

A consensual decision was reached to categorise injury and illness incidents according to the onset, in contrast to the traditional diagnostic classification of acute versus chronic progress. A sudden onset incident refers to an episode where the experienced distress or disability developed during minutes, seconds or less, while a gradual onset incident refers to an episode that developed during hours, days or more. The consensus group proposed that recordable incidents should be classified as follows:

- Sudden onset incident refers to a condition resulting from a specific identifiable episode resulting in a rapid onset of experienced distress or disability. Subsequently, sudden onset injuries can be categorised according to the cause of incident in:

- Traumatic injuries defined as a condition caused by an identifiable single external transfer of energy. Examples of traumatic sudden onset injuries include a bone fracture caused by a fall or a ligament tear caused by contact with an obstacle.

- Overuse injuries referring to a condition to which no identifiable single external transfer of energy can be associated. Multiple accumulative bouts of energy transfer could result in this kind of injury. Examples of overuse sudden onset injuries include tendon tears.

- Gradual onset incident refers to a condition that manifests itself over a period of time, or when there is a gradual increase in the intensity of experienced distress or disability, without a single identifiable event being responsible for the condition. Examples of gradual onset conditions include overtraining syndromes and overuse injuries, such as tendinosis/tendinopathies.

\section{Athlete baseline information}

Baseline information should include an athlete's age, gender, height, weight dominant arm/leg, main event(s), training volume (hours, sessions/week) and intensity, level of competition, number of years in Athletics and previous and ongoing incident(s). These two later need a detailed description as to when they happen, time loss, severity, injury body part, type, mode of onset, causes, contributing factors, if full recovery has been achieved, etc (see above details of recordable incidents). The categorisation of Athletics events should follow the groups proposed in box 5. Marathon could be combined with halfmarathon and road races, and hurdles with sprints.

\section{Study design, data collection procedures and analysis Study design and implementation}

Epidemiological studies in Athletics should ideally follow a prospective cohort design and record injury and illness incidence, thus allowing determination of risk factors. Cross-sectional study designs using prevalence measures are valuable for describing the burden of long-term, particularly overuse-related, conditions. Study designs involving repeated cross-sectional data collection can be used to establish prevalence trends. Any study must be approved by a recognised institutional (ethics) review board. It is recommended that studies are approved and endorsed by the Athletics organisations involved with the study population. Each athlete should be informed in writing about what is expected from her/him, the duration of the study, the risks and benefits of study participation and the procedures for the reporting of results (individually or at group level).

Table 2 Modes of onset, causes of injuries and contributing factors (modified from references 1, 2 and 18)

\begin{tabular}{|c|c|c|}
\hline Mode of onset & Causes & Contributing factors \\
\hline 1. Sudden onset incident & 1. Traumatic injury & A. Recurrence of previous injury \\
\hline \multirow[t]{6}{*}{ 2. Gradual onset incident } & 1.1. Contact injury & B. Violation of rules (obstruction, pushing) \\
\hline & 1.1.2. Contact with another athlete & C. Field of play conditions \\
\hline & 1.1.3. Contact: moving object (eg, discus) & D. Weather condition \\
\hline & 1.1.4. Contact: immobile object (eg, hurdles) & E. Equipment failure \\
\hline & 1.2. Non-contact injury & F. Fatigue \\
\hline & 2. Overuse injury & G. Psychological \\
\hline
\end{tabular}


Box 3 Illness-affected system (modified from references 2, 5,19 and 20)

1. Upper respiratory tract (nose, sinuses, pharynx, larynx)

2. Lower respiratory tract (trachea, bronchi, lungs)

3. Gastrointestinal

4. Cardiovascular

5. Urogenital, gynaecological or reproductive

6. Endocrine or metabolic

7. Haematological or immunological

8. Neurological, central nervous system

9. Dermatological/skin

10. Musculoskeletal

11. Dental

12. Ophthalmological/otological

13. Psychiatric/psychological

14. Other

Data collection in prospective studies should ideally be performed on a weekly basis. Motivating the reporting physicians, physiotherapists or athletes to consistently report in longitudinal studies is a key issue. Discontinued reporting from a participant should trigger an alarm generating a contact with the physician/ physiotherapist/athlete to encourage participation and to determine whether she/he is able to continue in the study. ${ }^{33}$ While it is highly recommended that injuries and illnesses in cohort studies are diagnosed and reported by qualified medical personnel, ${ }^{14}{ }^{15}$ prediagnostic data on injury and illness can also be collected by athlete self-reports. ${ }^{6}{ }^{12}$ The recording and reporting process should, if possible, be electronically managed and the system compliant with the guidelines of the Food and Drug Administration (FDA) in the USA (FDA 21 CFR Part 11). ${ }^{34}$ If computer-based tools are used for recoding of data, the electronic forms should be individually tested, even if the corresponding paper-based forms have been validated.

Prevalence trends can be monitored using studies with a panel, or if not possible, cross-sectional design, where athletes are asked to complete injury and illness questionnaires at regular time intervals, preferably each week. The Oslo Sports Trauma Research Centre (OSTRC) Questionnaire on Health Problems

Box 4 IIlness symptoms (modified from references 2, 5, 19 and 20)

1. Pain, ache or soreness

2. Fever, excess sweating or chills

3. Nausea, vomiting or diarrhoea

4. Weight loss or dehydration

5. Fatigue, lack of energy, lethargy or arterial hypotension

6. Irregular heartbeat, palpitation, syncope, collapse or chest pain

7. Congestion, hypersecretion rhinorrhoea or discharge

8. Cough, wheezing, dyspnoea or shortness of breath

9. Dizziness or vertigo

10. Rash, itch or eczema

11. Numbness, weakness or tingling

12. Mood/sleep disturbance, anxious or depressed

13. Other can be used for this purpose, ${ }^{23}{ }^{24}$ which focuses on four key questions on injury and illness with regard to: (1) consequences on sports participation; (2) consequences on training volume; (3) consequences on sports performance and (4) the degree to which the athlete has experienced symptoms.

\section{Exposure}

Athletic exposure divided into training and/or in competition should be reported in all studies. Exposure can be recorded based on time (eg, in training) or on participations/start (eg, in competition).

Competition exposure is defined as: "Competition including warm-up, the interval between starts during a competition day, competition and cool-down."

Training exposure is defined as: "Bodily movements or isometric force produced by skeletal muscles requiring energy expenditure and aimed at maintaining or improving an athlete's athletic performance." This includes skills, capabilities (power, speed, stamina), strength and conditioning and cross training (eg, running, cycling and swimming). Training volume is defined as the number of trained hours per week. Intensity of training can be assessed by using different intensity zones or effort scales per training session. ${ }^{35}$ By combining weekly hours and intensity of training per event, a training load rank index can be computed. ${ }^{6} 35$ Exercise and social sports activities that are not scheduled as part of an athlete's formal training programme should not be included as training exposure. If the aim of a study is to evaluate the incidence of injury associated with specific conditions (eg, training activities and capabilities), then the exposure to these specific conditions has to be recorded.

\section{Calculation of prevalence and incidence}

Incidence (requiring that the study population is 'healthy' with regard to the condition under study at the start of the data collection) is used to report findings from traditional prospective studies and prevalence is used to report long-term conditions in cross-sectional studies. Prospective studies based on panel designs use serial and average measures of prevalence to describe the magnitude of injury and illness. ${ }^{23} 24$

Prevalence is calculated by dividing the number of athletes with physical or psychological problems at/during a defined time period by the total number of athletes.

Incidence is calculated as number of new injuries or illnesses in relation to exposure (see above). Incidence should be calculated separately for illnesses, training injuries and in-competition injuries.

The overall incidence should be calculated as number of new injuries or illnesses recorded during the study period divided by a total athlete exposure hours (per $1000 \mathrm{~h}$ of athletic practice) or number of athletes (per 1000 athletes). The total athlete exposure hours is computed as the sum of recorded hours of training and competition during the period of the study.

Incidence of training injuries should be calculated as the number of new training injuries per $1000 \mathrm{~h}$ of training for Athletics. If the aim of a study is to measure the incidence of an injury associated with specific training activities, then the incidence of training injuries should be reported as a function of the athletes' exposure to the specific training activities; for example, the number of injuries during technical training (running/hurdles technique, jumps, throws, etc) per 1000 athlete-hours of technical training.

Incidence of in-competition injuries should be calculated as the number of new injuries in competition per 1000 registered athletes, per 1000 competing athletes and/or per 1000 athlete 
Table 3 Mode of onset and causes of illness (modified from references 2, 5, 19 and 20)

\begin{tabular}{|c|c|c|}
\hline Mode of onset & Causes of sudden illness & Causes of gradual onset illness \\
\hline \multirow[t]{8}{*}{$\begin{array}{l}\text { 1. Sudden onset incident } \\
\text { 2. Gradual onset incident }\end{array}$} & $\begin{array}{l}\text { 1.1. Pre-existing disease (exacerbations of allergy, asthma, diabetes, } \\
\text { degenerative, systemic inflammatory disorders, congenital, etc) }\end{array}$ & $\begin{array}{l}\text { 2.1. Pre-existing disease (exacerbations of allergy, asthma, diabetes, } \\
\text { degenerative, systemic inflammatory disorders, congenital, etc) }\end{array}$ \\
\hline & 1.2. Infectious (viral, bacterial, fungal, etc) & 2.2. Infectious (viral, bacterial, fungal, etc) \\
\hline & 1.3. Environmental (heat, cold, altitude, etc) & 2.3. Environmental (heat, cold, altitude, etc) \\
\hline & 1.4. Nutritional, endocrine or metabolic disturbance & 2.4. Nutritional, endocrine or metabolic disturbance \\
\hline & 1.5. Drug related or toxic reaction & 2.5. Drug related or toxic reaction \\
\hline & 1.6. Exercise related (dehydration, exhaustion, etc) & 2.6. Exercise related (dehydration, exhaustion, etc) \\
\hline & 1.7. Psychiatric & 2.7. Psychiatric \\
\hline & 1.8. Other/idiopathic & 2.8. Other/idiopathic \\
\hline
\end{tabular}

participations. $^{1} 18$ Registered athletes have been previously defined as all athletes officially registered for the competition by the governing body (entry list), independent of whether or not they participate in any competition. ${ }^{18}$ These include athletes who do not start and athlete who do not come to the competition. ${ }^{18}$ The number of competing athletes is defined as all athletes who start at least once in an event, irrespective of whether or not the athlete finishes the event. Athletes who do not finish (DNF), who are disqualified (DQ) or whose result is not measured (NM) are counted as having started. ${ }^{18}$ The number of athlete participations (or number of starts) is calculated by adding up the number of athletes starting in all heats, rounds, qualifications and finals of a particular discipline or day, including DNF, DQ and NM. ${ }^{18}$ If an athlete starts more than once in

Box 5 Athletic event groups (modified from references 1,2 and 32)

1. Sprints $(60,100,200$ and $400 \mathrm{~m})$ and relays $(4 \times 100$ and $4 \times 400)$

2. Middle distance runs $(800-1500 \mathrm{~m})$

3. Long-distance runs $(3000-10000 \mathrm{~m})$ including steeplechase (2000 and $3000 \mathrm{~m}$ steeplechase)

4. Cross-country races

5. Marathon, half-marathon and road races

6. Race walking $(5,10,20$ and $50 \mathrm{~km})$

7. Hurdles $(60,100,110$ and $400 \mathrm{~m}$ hurdles)

8. Jumps (high, long, triple and pole vault)

9. Throws (discus, javelin, hammer and shot put)

10. Combined events (pentathlon, heptathlon, octathlon and decathlon)

Notes: (1) This event group classification is based on the Athletics Olympic programme and also includes all official events at different official International Association of Athletics Federation (IAAF) Championships (indoors, outdoors, age groups, cross country, road races and race walking); (2) on previous Athletics studies, ${ }^{1} 2332$ marathon was separated from other long-distance runs as the distance is well enough defined for purposes of between-study comparisons; half-marathon and road races can be added in this group depending of the study design and studied populations; (3) hurdles may in some studies assemble small populations and can be added to sprints group; (4) ultra running, mountain running and other extreme distance road races were not included in this classification of event categories but could make up further athletic event groups and (5) cross-country race can be separated from the long-distance runs for comparison with other cross-country studies. the same or a different discipline, each start is counted. In combined disciplines (eg, heptathlon), the starts in each subdiscipline are counted. ${ }^{18}$

Incidence of in-competition illnesses should be calculated as the number of new illnesses per 1000 registered athletes or per 1000 competing athletes. ${ }^{18}$ To compare different championships, the incidence of in-competition illnesses can also be reported as new illnesses per 1000 athlete days. ${ }^{3}{ }^{36-38}$ The number of athlete days is calculated by multiplying the number of championship days by the number of registered athletes.

\section{Statistical analysis}

Incidence data should preferably be analysed with models based on the Cox proportional hazards regression method. Intrasubject correlations might be considered. ${ }^{39}$ Statistical models which take into account the complexity of competing events, repeated injuries, such as marginal methods, and multistate models with competing risk components are recommended. ${ }^{40}$ Different analysis for part-time loss and full-time loss may be considered. In addition, analysis of time between incidences has a substantial scientific value.

Serial measures of prevalence should be analysed using statistical models that account for the correlation of repeated outcome measures within participants over time, for example, generalised estimating equations or mixed-models.

\section{Reporting of results}

The presentation of the results should follow the 'STrengthening the Reporting of OBservational studies in Epidemiology' (STROBE) protocol for reporting of observational epidemiological studies (http://www.strobe-statement.org). ${ }^{41-43}$ The study population should be clearly defined with the number of participants, their age (mean and range), gender, main event and level of performance. The data should be reported in detail, including comments on the possible effects of missing data on the study results.

If the sample size is large enough, results should be reported separately for men and women, events, age categories and, if possible for ethnic group, country or geographical area (continents). For Athletics, athletes are divided into the following age categories: master (over 35years), senior (from 23 to 35 years), under 23 years, juniors (under 20 years) and youth (under 18 years). The circumstances (competition heat, round, final or training), severity (time loss), mode of onset, body location, side, type and causes should be accurately reported.

\section{CONCLUSION}

This consensus statement provides definitions and methodological guidance for the consistent recording and reporting on injuries and illness data in Athletics. Suggestions are made for 
recording athletes' baseline information, and competition and training exposures. Guidelines are presented on how incidents should be calculated and reported. Consistent use of the definitions and improved methodological guidance would lead to more reliable and comparable evidence.

\section{What is already known on this topic?}

- The current scientific literature on Athletics injury and illness epidemiology provides information from short-term surveillance during competitions and is limited to prospective studies covering the entire competitive season, possibly due to the unique challenges in conducting long-term prospective injury and illness surveillance in individual sports.

- Consensus statements on sports injury definitions and data collection procedures have been published for several team sports, and recently for individual sports.

- It is essential for Athletics to reach a similar agreement on injury and illness definitions and to use a standardised methodological approach to data collection to facilitate comparison and cumulative study.

\section{What this study adds?}

- This consensus statement provides definitions and methodological guidance for epidemiological studies in Athletics.

- Consistent use of the definitions and methodological guidance would lead to more reliable and comparable evidence.

\section{Author affiliations}

'Department of Medical and Health sciences, Linköping University, Linköping, Sweden

${ }^{2}$ International Association of Athletics Federations (IAAF) Medical and Anti-doping Commission, Montecarlo, Monaco

${ }^{3}$ Sports Medicine Department, Aspetar, Qatar Orthopedics and Sports Medicine Hospital, Doha, Qatar

${ }^{4}$ Swedish Athletics Association, Stockholm, Sweden

${ }^{5}$ FIFA Medical Assessment and Research Centre (F-MARC), Zurich, Switzerland

${ }^{6}$ Schulthess Klinik, Zurich, Switzerland

${ }^{7}$ Medical School Hamburg (MSH), Hamburg, Germany

${ }^{8}$ European Athletics Medical \& Anti-Doping Commission, European Athletics

Association (EAA), Lausanne, Switzerland

${ }^{9}$ Oslo Sports Trauma Research Centre, Oslo, Norway

${ }^{10}$ Olympic Elite Sports Program (Olympiatoppen), Oslo, Norway

${ }^{11}$ Diamond League, Stockholm, Sweden

${ }^{12}$ Karolinska Institutet, Stockholm, Sweden

${ }^{13}$ International Olympic Committee (IOC) Medical Commission, Lausanne, Switzerland

${ }^{14}$ Department of Sports Medicine, FINA Bureau, Lausanne, Switzerland

${ }^{15}$ McMaster University School of Medicine, Hamilton, Ontario, Canada

${ }^{16}$ Royal Netherlands Lawn Tennis Association, Amersfoort, The Netherlands

${ }^{17}$ Department of Clinical and Exercise Physiology, Sports Medicine Unit, Faculty of Medicine, University Hospital of Saint-Etienne, Saint-Etienne, France

${ }^{18}$ Laboratory of Exercise Physiology (LPE EA 4338), University of Lyon, Saint-Etienne, France

${ }^{19}$ Medical Commission, French Athletics Federation (FFA), Paris, France

Contributors TT, J-MA, JJ, AJ and PE made substantial contributions to conception and design of the project; meeting participation; drafting the article and revising it critically for important intellectual content and final approval of the version to be published. $\mathrm{PB}, \mathrm{BC}, \mathrm{JK}, \mathrm{MM}, \mathrm{SN}, \mathrm{BP}, \mathrm{PR}, \mathrm{OR}$ and $\mathrm{KS}$ participated in the consensus meeting; gave substantial contributions to critically revising the article for important intellectual content; revised the English language presentation (MM) and final approval of the version to be published.

Funding Supportive funding to cover meeting expenses for consensus group members was obtained from Linköping University and the Swedish Athletics Association.

\section{Competing interests None.}

Provenance and peer review Not commissioned; externally peer reviewed.

\section{REFERENCES}

1 Alonso JM, Junge A, Renström P, et al. Sports injuries surveillance during the 2007 IAAF World Athletics Championships. Clin I Sport Med 2009;19:26-32.

2 Alonso JM, Tscholl PM, Engebretsen L, et al. Occurrence of injuries and illnesses during the 2009 IAAF World Athletics Championships. Br I Sports Med 2010;44:1100-5

3 Alonso JM, Edouard $P$, Fischetto $G$, et al. Determination of future prevention strategies in elite track and field: analysis of Daegu 2011 IAAF Championships injuries and illnesses surveillance. Br J Sports Med 2012;46:505-14.

4 Junge A, Engebretsen L, Mountjoy ML, et al. Sports injuries during the Summer Olympic Games 2008. Am J Sports Med 2009:37:2165-72.

5 Engebretsen L, Soligard T, Steffen K, et al. Sports injuries and illnesses during the London Summer Olympic Games 2012. Br I Sports Med 2013;47:407-14.

6 Jacobsson J, Timpka T, Kowalski J, et al. Injury patterns in Swedish elite athletics: annual incidence, injury types and risk factors. Br J Sports Med 2013;47:941-52.

7 Bennell KL, Crossley K. Musculoskeletal injuries in track and field: incidence, distribution and risk factors. Aust I Sci Med Sport 1996;28:69-75.

8 D'Souza D. Track and field athletics injuries-a one-year survey. Br J Sports Med 1994;28:197-202.

9 Edouard P, Depiesse F, Hertert P, et al. Injuries and illnesses during the 2011 Paris European Athletics Indoor Championships. Scand J Med Sci Sports 2013;23:e213-18.

10 Edouard P, Depiesse F, Branco P, et al. Analyses of Helsinki 2012 European Athletics Championships injury and illness surveillance to discuss elite athletes risk factors. Clin I Sport Med 2013 [Epub ahead of print].

11 Lysholm J, Wiklander J. Injuries in runners. Am J Sports Med 1987;15:168-71.

12 Jacobsson J, Timpka T, Kowalski J, et al. Prevalence of musculoskeletal injuries in Swedish elite track and field athletes. Am I Sports Med 2012:40:163-9.

13 Rauh MJ, Koepsell TD, Rivara FP, et al. Epidemiology of musculoskeletal injuries among high school cross-country runners. Am J Epidemiol 2006;163:151-9.

14 Fuller CW, Ekstrand J, Junge $A$, et al. Consensus statement on injury definitions and data collection procedures in studies of football (soccer) injuries. Clin I Sport Med 2006;16:97-106

15 Fuller CW, Molloy MG, Bagate C, et al. Consensus statement on injury definitions and data collection procedures for studies of injuries in rugby union. Clin I Sport Med 2007;17:177-81.

16 Pluim BM, Fuller CW, Batt ME, et al. Consensus statement on epidemiological studies of medical conditions in tennis, April 2009. Br J Sports Med 2009:43:893-7.

17 Turner M, Fuller CW, Egan D, et al. European consensus on epidemiological studies of injuries in the thoroughbred horse racing industry. Br J Sports Med 2012:46:704-8.

18 Junge $\mathrm{A}$, Engebretsen $\mathrm{L}$, Alonso JM, et al. Injury surveillance in multi-sport events: the International Olympic Committee approach. Br J Sports Med 2008:42:413-21.

19 Engebretsen L, Steffen K, Alonso JM, et al. Sports injuries and illnesses during the Winter Olympic Games 2010. Br J Sports Med 2010;44:772-80.

20 Mountjoy M, Junge A, Alonso JM, et al. Sports injuries and illnesses in the 2009 FINA World Championships (Aquatics). Br I Sports Med 2010:44:522-7.

21 Orchard J, Hoskins W. For debate: consensus injury definitions in team sports should focus on missed playing time. Clin I Sport Med 2007:17:192-6.

22 Bahr R. No injuries, but plenty of pain? On the methodology for recording overuse symptoms in sports. Br J Sports Med 2009;43:966-72.

23 Clarsen B, Rønsen O, Myklebust G, et al. The Oslo Sports Trauma Research Center questionnaire on health problems: a new approach to prospective monitoring of illness and injury in elite athletes. Br I Sports Med Published Online First: 21 Feb 2013. doi:10.1136/bjsports-2012-092087

24 Clarsen B, Myklebust G, Bahr R. Development and validation of a new method for the registration of overuse injuries in sports injury epidemiology. $\mathrm{Br} /$ Sports Med 2013:47:495-502.

25 Hammond LE, Lilley JM, Ribbans WJ. Defining recovery: an overlooked criterion in sports injury surveillance. Clin I Sport Med 2013;23:157-9.

26 Fuller CW, Bahr R, Dick RW, et al. A framework for recording recurrences, reinjuries, and exacerbations in injury surveillance. Clin I Sport Med 2007:17:197-200.

27 Finch CF, Cook J. Categorising sports injuries in epidemiological studies: the subsequent injury categorisation $(\mathrm{SIC})$ model to address multiple, recurrent and exacerbation of injuries. Br J Sports Med Published Online First: 16 Mar 2013. doi:10.1136/bjsports-2012-091729 
28 Rae K, Orchard J. The Orchard Sports Injury Classification System (OSICS) version 10. Clin I Sport Med 2007;17:201-4.

29 Meeuwisse WH, Wiley JP. The sport medicine diagnostic coding system. Clin I Sport Med 2007;17:205-7.

30 WHO. International Statistical Classification of Diseases and Related Health Problems. 10th revision, WHO, 1999.

31 WHO. International Classification of Functioning, Disability and Health (ICF). WHO, 2001.

32 Feddermann $N$, Junge $A$, Branco $P$, et al. Injuries in 13 international Athletics championships between 2007-2012. Br J Sports Med 2014;48:513-22.

33 Jacobsson J, Timpka T, Ekberg J, et al. Design of a protocol for large-scale epidemiological studies in individual sports: the Swedish Athletics injury study. $\mathrm{Br} J$ Sports Med 2010;44:1106-11

34 FDA. CFR—Code of Federal Regulations Title 21, 2013.

35 Foster C, Florhaug JA, Franklin J, et al. A new approach to monitoring exercise training. J Strength Cond Res 2001;15:109-15.

36 Dvorak J, Junge $A$, Derman W, et al. Injuries and illnesses of football players during the 2010 FIFA World Cup. Br J Sports Med 2011;45:626-30.

37 Derman W, Schwellnus $\mathrm{M}$, Jordaan $\mathrm{E}$, et al. Illness and injury in athletes during the competition period at the London 2012 Paralympic Games: development and implementation of a web-based surveillance system (WEB-IISS) for team medical staff. Br J Sports Med 2013;47:420-5.

38 Schwellnus M, Derman W, Jordaan E, et al. Factors associated with illness in athletes participating in the London 2012 Paralympic Games: a prospective cohort study involving 49910 athlete-days. Br J Sports Med 2013:47:433-40.

39 Hamilton GM, Meeuwisse WH, Emery CA, et al. Past injury as a risk factor: an illustrative example where appearances are deceiving. Am J Epidemiol 2011;173:941-8.

40 Lawless JF, Cook RJ. eds The statistical analysis of recurrent events. New York, NY: Springer Verlag, 2007.

41 Langan SM, Benchimol El, Guttmann A, et al. Setting the RECORD straight: developing a guideline for the REporting of studies Conducted using Observational Routinely collected Data. Clin Epidemiol 2013;5:29-31.

42 Vandenbroucke JP, von Elm E, Altman DG, et al. Strengthening the Reporting of Observational Studies in Epidemiology (STROBE): explanation and elaboration. Epidemiology 2007:18:805-35.

43 von Elm E, Altman DG, Egger M, et al. The Strengthening the Reporting of Observational Studies in Epidemiology (STROBE) statement: guidelines for reporting observational studies. Epidemiology 2007;18:800-4. 